\title{
ADAPTACIÓN Y VALIDACIÓN DE LA ESCALA DE NOMOFOBIA DE YILDIRIM Y CORREIA EN ESTUDIANTES ESPAÑOLES DE LA EDUCACIÓN SECUNDARIA OBLIGATORIA
}

\author{
ADAPTATION AND VALIDATION OF THE YILDIRIM \& CORREIA \\ NOMOPHOBIA SCALE IN SPANISH STUDENTS OF 'COMPULSORY \\ SECONDARY EDUCATION'
}

Irene Ramos-Soler, Carmen López-Sánchez y Mª Carmen Quiles-Soler

Universidad de Alicante

\section{Abstract}

Although is not a recent phenomenon, Nomophobia (No Mobile Phone Phobia), understood as a behavioral addiction that implies a high fear of being left without a mobile phone, is being researched from different disciplines given the important consequences that are being observed between the younger population. However, there are few instruments to measure this addiction not recognized by the DSM-V. In the present work the process of adaptation and validation of the measurement scale of the Nomophobia of Yildirim and Correia has been carried out. A linguistic and cultural adaptation of the scale was carried out, followed by a review by the experts in the field. The group of experts proposed a modification of the wording and the inclusion of a new item. The final scale was applied to a sample of 372 students of Compulsory Secondary Education, in public institutes of the Province of Alicante. The results allowed the validation of the Yildirim and Correia scale.

Keywords: social networks, smartphone, nomophobia, teenagers, addiction, scale validation.

\section{Resumen}

Aunque no se trata de un fenómeno reciente, la Nomofobia (No Mobile Phone Phobia) entendida como una adicción comportamental que implica un alto temor a quedarse sin teléfono móvil, está siendo objeto de investigación desde diferentes disciplinas dada las importantes consecuencias que se están observando entre la población más joven. Sin embargo, son pocos los instrumentos capaces de medir esta adicción no reconocida como tal por el DSM-V. En el presente trabajo se ha llevado a cabo el proceso de adaptación y validación de la escala de medición de la Nomofobia de Yildirim y Correia. Para ello se procedió a una adaptación lingüística y cultural de la misma, seguida de una revisión por parte de expertos en la materia. La decisión del grupo de expertos supuso una modificación de la redacción y la inclusión de un nuevo ítem. La escala resultante se aplicó a una muestra de 372 estudiantes de Educación Secundaria Obligatoria, en institutos públicos de la provincia de Alicante. Los resultados permitieron la validación de la escala objeto de este estudio.

Palabras clave: redes sociales, smartphone, nomofobia, adolescentes, adicción, validación de escala. 
De acuerdo con el último estudio publicado por Digital Marketing Trends (Ditrendia, 2016), a finales del año 2015 el 97\% de la población mundial utilizaba el teléfono móvil y el número de dispositivos superaba el de personas en nuestro planeta. Si observamos los datos relativos a España, cabe resaltar el hecho de que el número de teléfonos móviles inteligentes supera al de los ordenadores (un $80 \%$ frente a un $73 \%$ ). En cuanto al sector de la población joven, este mismo estudio muestra como el 98\% de los jóvenes de entre 10 y 14 años disponía de un teléfono móvil con acceso a Internet.

Desde otro punto de vista, atendiendo al uso que se da a los teléfonos móviles, el mismo estudio afirma que en España su uso gira en torno a $3 \mathrm{~h}$ y $23 \mathrm{~m}$ a lo largo del día, mientras que el uso de aplicaciones asciende al 54\% del tiempo invertido en el ámbito virtual.

En este artículo se plantea el uso que los adolescentes hacen del teléfono móvil en el entorno virtual y, más concretamente, en el de las redes sociales. En este sentido podemos afirmar, el $41 \%$ de los españoles accede a sus redes sociales utilizando el móvil como dispositivo de conexión, siendo la población joven la que más simpatiza con ellas (Ditrendia, 2016). Son muchas las redes sociales que la población en general tiene a su disposición: Facebook, WhatsApp, Twitter, Instagram, Youtube... las cuales han tenido una gran penetración en nuestra sociedad. No obstante, no todas tienen la misma valoración. De acuerdo con los datos extraídos del último Estudio Anual de Redes Sociales difundido por la Interactive Advertising Boureau (IAB, 2016), las redes sociales que han alcanzado una mejor puntuación son WhatsApp y YouTube, seguidas de Spotify, Instagram, Telegram y Facebook. Mientras que entre las peor valoradas sobresalen Google+, Line y LinkedIn.

Por otra parte, el uso que se da a cada una de ellas también es variable y abarca desde las $5 \mathrm{~h} 14 \mathrm{~min} / \mathrm{semana}$ destinadas a Whatsapp o las 4h24min/semana a Spotiffy, a la 1h23min que se dedica a Linkedln. Siguiendo con el mismo informe (IAB, 2016) cabe resaltar que el carácter social de estas redes sigue siendo una realidad, lo que confirma el hecho de que chatear, o ver qué hacen los contactos, sigue siendo el principal cometido de éstas, seguido por ver vídeos o escuchar música.

Todas estas cifras nos llevan a plantear la evidencia de que la población en general, y los adolescentes en particular está altamente condicionada en su vida cotidiana por Internet y las redes sociales. Tal es la realidad en la que vivimos que se plantea el hecho de que algunos individuos han desarrollado conductas adictivas hacia Internet (Christakis et al., 2011) y hacia las redes sociales. En este sentido, como sucede cuando hablamos de consumos de sustancias que pueden generar adicción como es el caso del alcohol, el problema no es tanto su uso sino su abuso. En el momento en el que se produce un abuso en el uso/consumo de Internet y de las Redes Sociales, los adolescentes como grupo de edad más vulnerable por sus procesos biológicos y sociales se sienten fuertemente atraídos hacía ellas y más expuestos a las consecuencias negativas que se pueden desprender del mismo. Tal y como afirman Gomes-Franco-e-Silva y Sendín-Gutiérrez (2016):

Los jóvenes constituyen el grupo más propenso al desarrollo de un uso problemático de la Red al encontrarse en una etapa crítica -adolescencia- de definición de su identidad. La inestabilidad emocional e inseguridad pueden motivarles a buscar refugio en la web y en las herramientas sociales on-line sin que los adultos apenas lo adviertan. (p.47)

Arab y Díez (2015) exponen algunos de los aspectos negativos vinculados al uso de las redes sociales. El Grooming, el Ciberbullying, el Sexting y la ciberadicción, son algunas de las prácticas o conductas nocivas que pueden llegar a desarrollar quiénes hacen un uso abusivo e inapropiado de las redes sociales.

Sin embargo, el uso de las redes sociales proporciona en ocasiones una serie de ventajas que no se deben obviar. Además de ayudar a ciertos colectivos con dificultades en su día a día, gracias a las redes sociales los jóvenes pueden mantener contacto con sus amistades de un modo instantáneo y económico (Espinar y González, 2009); les proporcionan autonomía y accesibilidad inmediata ya que sólo necesitan una conexión a Internet (Suriá, 2015). Por otra parte, la creación de blogs o vídeos pueden favorecer la competencia, mientras que permiten generar elementos con contenido educativo, así como colaborar en la educación sexual o en la promoción y prevención en el ámbito de la salud, entre otras cuestiones (Arab y Díez, 2015).

Pero no sólo preocupa la adicción que jóvenes y adolescentes pueden llegar a desarrollar hacia las redes sociales. Más allá de estos entornos virtuales el 
dispositivo de acceso a los mismos se convierte en un claro mediador entre éstos y sus experiencias en la red, experiencias que suelen alcanzar gracias a la conexión a través de sus smartphones. Es precisamente esa necesidad de estar constantemente conectados lo que hace que jóvenes y adolescentes se sientan altamente dependientes de ellos. Sin embargo, de acuerdo con Simó, Martínez, Ballester y Domínguez (2017) no existe un fundamento teórico sólido que sostenga este trastorno de la conducta ni instrumentos de evaluación elaborados a partir de criterios unificados. Estos autores, a partir de una revisión de las escalas de medición sobre el uso del móvil publicadas en diferentes bases de datos, observaron que es necesario "utilizar una taxonomía única y exacta que permita interpretar y comparar los resultados de los distintos estudios" (Simó et al., 2017, p.12).

Algunas de las denominaciones acuñadas para hablar de este problema son las de uso problemático del móvil y/o uso dependiente del móvil ya que "existe todavía una gran controversia sobre la evidencia de adicciones conductuales en el marco de las tecnologías de información y comunicación" (Tresáncoras, GarcíaOliva, y Piqueras, 2017, p.28). Sin embargo parece que se está generalizando un concepto que delimitaría de manera clara y precisa la situación a la que nos enfrentamos: la Nomofobia (No Mobile Phone Phobia).

La nomofobia podría definirse como "el miedo a quedarse fuera de contacto con el móvil" (SecurEnvoy, 2012). King, Valença, y Nardi (2010) se refieren a ella como "un temor a convertirse tecnológicamente incomunicado, distante del teléfono móvil o no conectado a la web" (p.58). Por lo tanto, de acuerdo con Bragazzi y del Puente (2014) se podría afirmar que quienes padecen nomofobia sienten un miedo irracional a salir de casa sin el teléfono móvil, sintiendo una gran ansiedad cuando pierden su móvil, se les agota la batería o no tienen cobertura ya que se sienten aislados de familiares y amigos, porque su trabajo les exige estar permanentemente conectados; o porque sienten la necesidad constante de estar enterado de lo que hacen los otros.

Sin embargo, y a pesar de la importancia que está adquiriendo este desorden entre la población más joven, apenas existen estudios que lo aborde. De hecho, uno de los problemas a los que nos enfrentamos a la hora de estudiar esta 'nueva adicción' es que "las adicciones comportamentales, a excepción del juego de azar, no se encuentran reconocidas en el DSM-V (Diagnostic and Statistical Manual)" (Simó Sanz et al., 2017, p.5). En este sentido, de las 21 escalas de medición revisadas por estos autores, ninguna hacía alusión explícita a la nomofobia.

Yildirim y Correia (2015) desarrollaron y validaron un cuestionario, compuesto por 20 ítems, para medir la nomofobia entre estudiantes universitarios en Estados Unidos. La fiabilidad del cuestionario se determinó estudiando su consistencia interna a partir del Alfa de Cronbach, el cual tomó un valor de 0.945. Dicho instrumento sigue una escala tipo Likert de siete puntos, en la que 1 significa "totalmente en desacuerdo" y 7 "totalmente de acuerdo". La población estadounidense sobre la que se aplicó el cuestionario puede tener rasgos culturales diferentes a la española, por ello, en este trabajo se plantea como objetivo principal realizar la adaptación transcultural y validación estadística de dicha escala y describir el uso y/o abuso de los smartphones en estudiantes de la Educación Secundaria Obligatoria (ESO).

\section{MÉTODO}

\section{Procedimiento}

Para adaptar el cuestionario a la muestra de estudiantes españoles y a los objetivos de nuestra investigación se procedió, en primer lugar, a la adaptación lingüística y cultural de la escala siguiendo las directrices de la Comisión Internacional de Tests (Hambleton, 1994). Tras la traducción de la misma, se inició un proceso de criba por parte de expertos en la materia, a los que se les envió el cuestionario junto a los objetivos y características de la investigación. Tras el dictamen del juicio de expertos se modificó su redacción de forma considerable, incluyéndose un ítem adicional. Se decidió reducir la escala tipo Likert de siete puntos, a cinco, donde 1 significaba "totalmente en desacuerdo y 5 "totalmente de acuerdo", con el objetivo de facilitar la respuesta de la población investigada cuya edad es inferior (12-16 años) a la original, compuesta por universitarios mayores de edad. Finalmente se llegó a la conclusión de que los enunciados propuestos eran correctos (ver Tabla 1). 
Tabla 1. Escala de Nomofobia Adaptada



Fuente: Elaboración propia

\section{Parcitipantes}

El cuestionario resultante se ha aplicado sobre una muestra de 372 estudiantes de Educación Secundaria Obligatoria, en institutos públicos, de la provincia de Alicante. El tamaño de la muestra se considera lo suficientemente amplio para la significación estadística de un análisis factorial exploratorio (Tabachnick \& Fidell, 2013; DeVellis, 2003; Comrey \& Lee, 1992). La muestra se distribuye de la siguiente manera, $53.2 \%$ son chicos y el $46.8 \%$ son chicas, en cuanto al nivel de estudios alcanzados un $35.8 \%$ está en primero de la ESO, el 33.7\% en segundo, el $10 \%$ en tercero y el $20.5 \%$ en cuarto. Para el tratamiento de los datos se ha utilizado el programa SPSS 15.0 y SPAD 5.0.

\section{RESULTADOS}

La consistencia de la escala de nomofobia original viene avalada por la revisión efectuada entre las principales aportaciones teóricas y empíricas sobre su medición (Yildirim y Correia, 2015; Walsh et al., 2010). Se procedió a medir su fiabilidad tras la adaptación a los estudiantes españoles de la ESO. En primer lugar, se estimó su consistencia interna a partir del coeficiente Alfa de Cronbach, el cual debe tomar un valor mínimo de 0.70 para afirmar que la escala multítem tiene consistencia interna y es fiable (Grande y Abascal, 2003). Como puede observarse en la tabla 2 que se presenta a continuación, el valor obtenido por el coeficiente Alfa de Cronbach es 0.903, indicando que el cuestionario tiene una buena consistencia interna. De hecho, un valor alfa de 0.903 se considera excelente (George y Mallery, 2011).

Para poder determinar de una forma más precisa la consistencia interna, se ha calculado de nuevo el coeficiente alfa incorporando en los descriptivos el valor que toma si se elimina cada ítem de la escala de forma independiente, el objetivo es ver si el alfa sube o baja para mejorar su consistencia. 
Tabla 2. Estadísticos de fiabilidad

\begin{tabular}{ccc}
\hline Alfa de Cronbach & Alfa de Cronbach basada en los elementos tipificados & 0.905 \\
\hline 0.903 & 21 \\
\hline Fuente: Elabormero de elementos
\end{tabular}

Fuente: Elaboración propia

Tabla 3. Estadísticos total-elemento

\begin{tabular}{|c|c|c|c|c|c|}
\hline & & $\begin{array}{l}\text { Media de la } \\
\text { escala si se } \\
\text { elimina el } \\
\text { elemento }\end{array}$ & $\begin{array}{l}\text { Varianza de la } \\
\text { escala si se } \\
\text { elimina el } \\
\text { elemento }\end{array}$ & $\begin{array}{c}\text { Correlación } \\
\text { elemento-total } \\
\text { corregida }\end{array}$ & $\begin{array}{c}\text { Alfa de } \\
\text { Cronbach si } \\
\text { se elimina el } \\
\text { elemento }\end{array}$ \\
\hline 1 & Cuando no tengo wifi en el móvil me pongo furioso & 46,4892 & 233,701 & ,537 & 899 \\
\hline 2 & Me molesto cuando quiero buscar algo desde mi móvil y no puedo & 45,9973 & 236,752 & ,429 & ,901 \\
\hline 3 & $\begin{array}{l}\text { A veces pienso que se me va a acabar la batería del móvil y me pongo } \\
\text { nervioso }\end{array}$ & 46,3280 & 228,943 &, 554 &, 898 \\
\hline 4 & $\begin{array}{l}\text { Si me quedo sin wifi, inmediatamente busco señal para conectarme donde } \\
\text { sea }\end{array}$ & 46,5188 & 231,523 &, 538 & 899 \\
\hline 5 & Me da mucho miedo quedarme sin datos en mi móvil & 46,8253 & 230,942 &, 578 & 898 \\
\hline 6 & Me siento seguro si no llevo el móvil conmigo & 46,3011 & 248,006 &, 082 & ,911 \\
\hline 7 & $\begin{array}{l}\text { Tengo la necesidad de estar mirando el móvil continuamente por si hay } \\
\text { algún mensaje nuevo }\end{array}$ & 46,3091 & 229,562 &, 556 & 898 \\
\hline 8 & $\begin{array}{l}\text { Me agobio mucho si no tengo el móvil y pienso que alguien quiere } \\
\text { comunicarse conmigo }\end{array}$ & 46,2312 & 225,305 & ,638 &, 896 \\
\hline 9 & Cuando no tengo el móvil no sé qué hacer & 46,6559 & 225,105 & 699 & 895 \\
\hline 10 & $\begin{array}{l}\text { Me despisto con las tareas del colegio/instituto por estar mirando y } \\
\text { utilizando mi móvil }\end{array}$ & 46,5081 & 231,916 &, 546 & 899 \\
\hline 11 & $\begin{array}{l}\text { Aunque quisiera no podría prescindir de mi móvil. Si me lo quitaran en casa } \\
\text { me pondría furioso }\end{array}$ & 46,3548 & 230,343 &, 542 & 899 \\
\hline 12 & Cuando me siento mal, comienzo a usar el móvil y me siento mucho mejor & 46,3414 & 227,358 &, 549 & 898 \\
\hline 13 & Por estar usando el móvil duermo menos & 46,3091 & 230,920 & ,493 &, 900 \\
\hline 14 & $\begin{array}{l}\text { Si me llaman o tengo un aviso en el móvil lo atiendo inmediatamente, } \\
\text { aunque esté con mi familia o amigos }\end{array}$ & 46,1371 & 227,860 &, 583 & 898 \\
\hline 15 & Suelo estar mirando el móvil aunque esté con gente, familia o amigos & 46,0914 & 228,412 & ,625 & 897 \\
\hline 16 & No puedo salir a la calle sin móvil, si se me olvida, vuelvo a casa a por él & 46,2097 & 232,182 & ,472 & 900 \\
\hline 17 & $\begin{array}{l}\text { Me disgusta tener que apagar el móvil en clase, en comidas familiares, en } \\
\text { actos sociales, cine, etc }\end{array}$ & 46,8065 & 233,127 &, 536 & 899 \\
\hline 18 & Me siento más feliz cuanto estoy usando el móvil & 46,4704 & 231,220 &, 541 & 899 \\
\hline 19 & $\begin{array}{l}\text { Sería la persona más feliz del mundo si me regalaran un móvil de última } \\
\text { generación }\end{array}$ & 46,1371 & 226,631 &, 582 & 898 \\
\hline 20 & $\begin{array}{l}\text { Los mayores se enfadan conmigo porque le presto más atención al móvil } \\
\text { que a ellos }\end{array}$ & 46,4409 & 227,040 & ,658 &, 896 \\
\hline 21 & $\begin{array}{l}\text { Mis amigos se enfadan conmigo porque le hago más caso al móvil que a } \\
\text { ellos }\end{array}$ & 47,1075 & 241,309 & ,393 & ,902 \\
\hline
\end{tabular}

Fuente: Elaboración propia

El valor del coeficiente alfa, si se eliminara el ítem correspondiente, en la mayoría de los casos, desciende, pero superando siempre el mínimo aceptado de 0.7, por lo que la presencia de todos ellos mejora la consistencia interna de la escala. El enunciado 6 es el único que muestra un resultado distinto, si se suprimiese, la consistencia global seria ligeramente superior, sin embargo, si se mantiene, el Alfa de Cronbach seguiría siendo excelente, por lo se ha decidido no eliminarlo. En la misma tabla se puede observar el valor de la 
correlación "elemento-total corregida", siendo este el coeficiente de homogeneidad corregido, vemos que no hay ningún valor cero o negativo, por lo que todos los enunciados correlacionan con el total.

Tabla 4. Análisis de Componentes principales. Varianza total explicada

\begin{tabular}{|c|c|c|c|c|c|c|}
\hline \multirow[b]{2}{*}{ Componente } & \multicolumn{3}{|c|}{ Autovalores iniciales (valores propios) } & \multicolumn{3}{|c|}{ Sumas de las saturaciones al cuadrado de la extracción } \\
\hline & Total & \% de la varianza & $\%$ acumulado & Total & $\%$ de la varianza & $\%$ acumulado \\
\hline 1 & 7.521 & 35.815 & 35.815 & 7.521 & 35.815 & 35.815 \\
\hline 2 & 1.410 & 6.717 & 42,531 & 1,410 & 6,717 & 42,531 \\
\hline 3 & 1,180 & 5,620 & 48,151 & 1,180 & 5,620 & 48,151 \\
\hline 4 & 1,113 & 5,302 & 53,453 & 1,113 & 5,302 & 53,453 \\
\hline
\end{tabular}

Fuente: Elaboración propia

Tabla 5. Análisis de componentes principales. Matriz de componentes

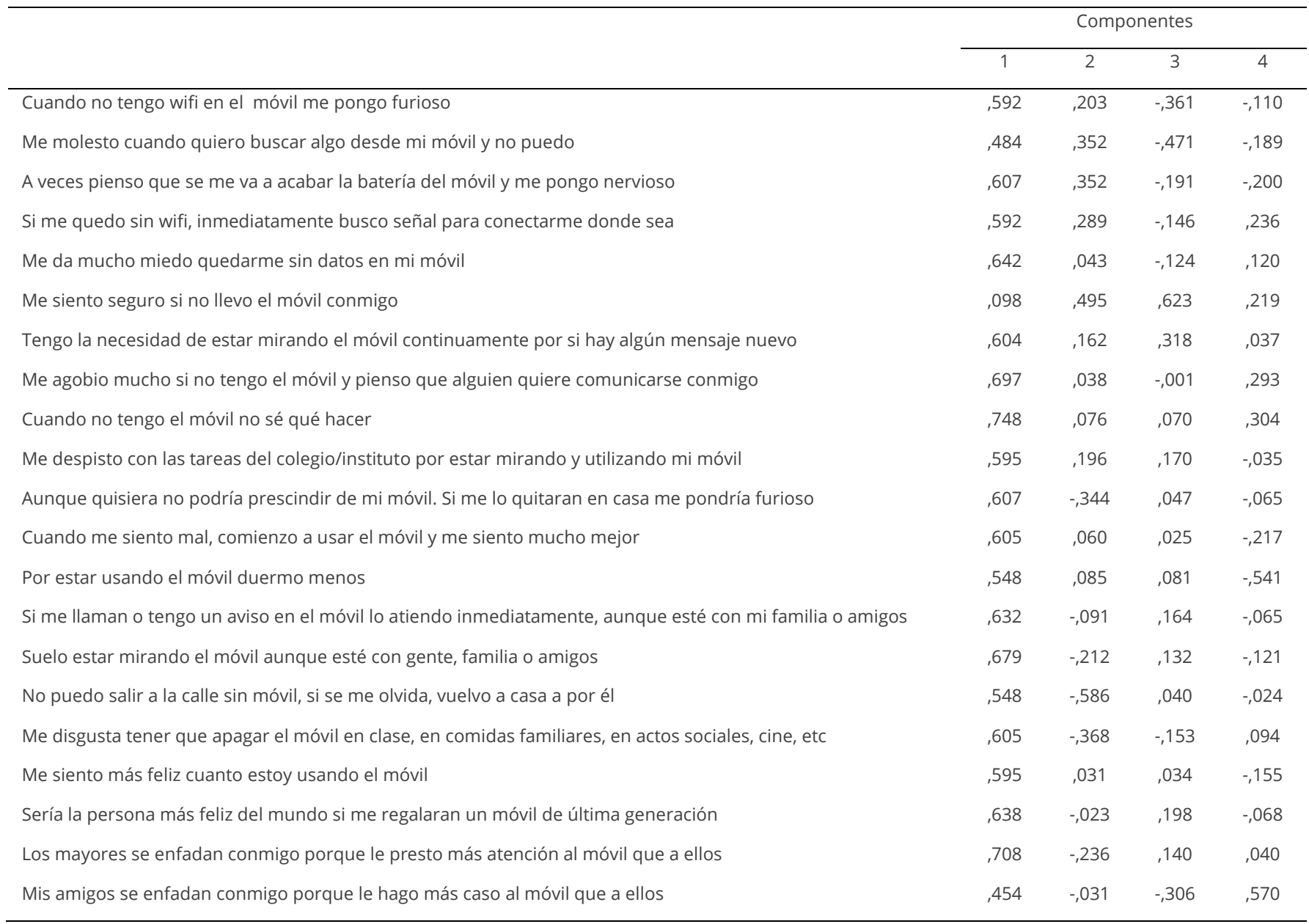

Fuente: Elaboración propia

Para validar la escala también se ha realizado un análisis factorial de componentes principales, con el objetivo de comprobar si los ítems se agrupaban en un solo componente, y contribuían así a explicar un mismo fenómeno, en este caso la nomofobia. En la escala original de Yildirim y Correia (2015) se identificaron cuatro dimensiones de la nomofobia, tras el análisis realizado en nuestra investigación cuatro son los 
componentes que explican el $53.5 \%$ de la varianza (Tabla 4), siendo sus valores propios superiores a 1. En cuanto a la matriz de componentes (Tabla 5), se observa como todos los ítems tienen sus pesos más elevados en torno al primer componente, obteniendo valores superiores a 0.45 en todos los casos, exceptuando de nuevo, el enunciado seis, que obtiene pesos mayores en los componentes 2 y 3 . Los resultados muestran en este sentido que todos los ítems tienen resultados relevantes alrededor del primer componente, que es el que más varianza explica, lo que unido al Alfa de Cronbach obtenido nos indican que estamos ante una escala válida y fiable para medir la nomofobia entre los adolescentes españoles.

\section{Nomofobia. Análisis factorial de componentes principales}

La respuesta dada por los estudiantes de la ESO a cada uno de los ítems de la escala de nomofobia validada, se ha utilizado para realizar un análisis factorial y de clasificación de componentes principales con el programa de análisis estadístico SPAD 5.0. El objetivo es medir el nivel de nomofobia y agrupar a los estudiantes en segmentos en función dicho resultado, describiendo los grupos obtenidos a partir de variables sociodemográficas y hábitos de consumo digital.

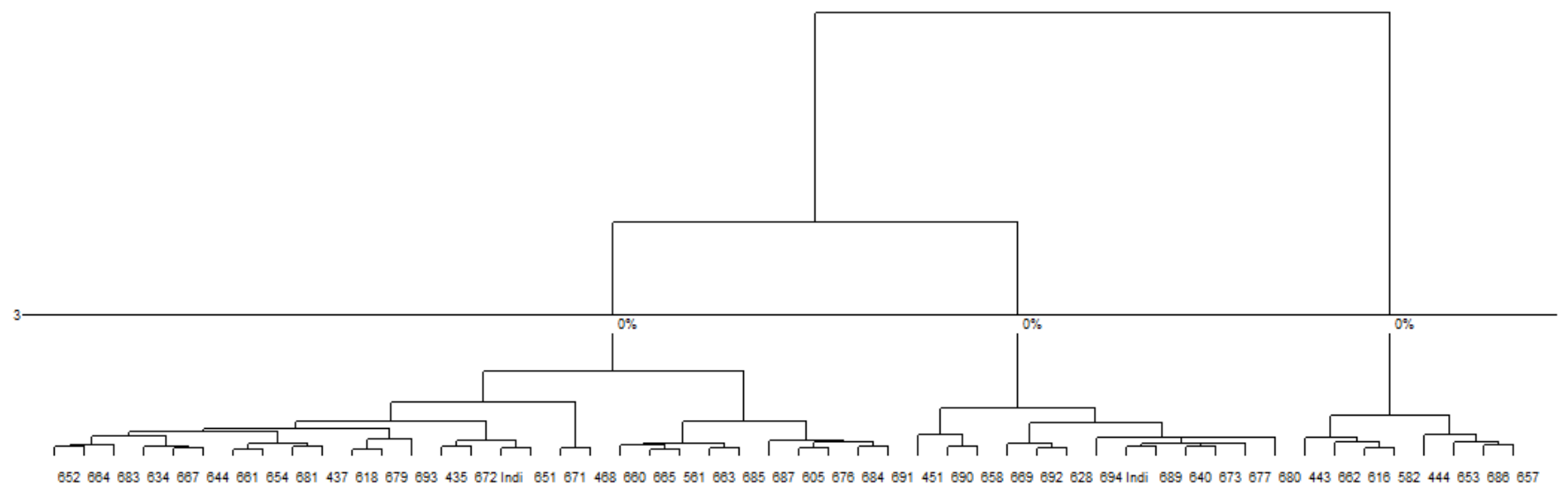

Figura 1. Dendograma de la clasificación

Esta técnica de análisis factorial analiza tablas de variables métricas. Para realizar el análisis se han seleccionado como variables activas la escala de nomofobia aplicada en la encuesta y como variables ilustrativas el resto: variables sociodemográficas, estilos de vida y consumo digital. El dendograma de la clasificación aparece en la Figura 1.

Tras observar el dendograma, se ha decidido obtener una partición en tres grupos o clases. Para ello se ha seguido el principio general del análisis de clasificación que busca que las variaciones internas de los clusters sean mínimas y una separación máxima entre grupos. El cuadro siguiente describe las inercias inter e intragrupos (ver Tabla 6).
Tabla 6. Descripción de las particiones en tres clases

\begin{tabular}{lcccc}
\hline & INERCIAS & EFECTIV. & PESOS & DISTANCIA \\
\hline $\begin{array}{l}\text { INERCIA } \\
\text { INTERGRUPOS }\end{array}$ & 6.4342 & & & \\
INERCIA & & & & \\
INTRAGRUPOS & & & & \\
Grupo 1/3 & 4.3808 & 152 & 152 & 0.8323 \\
Grupo 2/3 & 3.3679 & 175 & 175 & 4.7568 \\
Grupo 3/3 & 1.8624 & 45 & 45 & 31.8789 \\
INERCIA TOTAL & 16.0453 & & & \\
\end{tabular}

Fuente: Elaboración propia

Los grupos 1 y 2 son los más numerosos, compuestos por 152 y 175 estudiantes respectivamente, siendo el más heterogéneo el primero ya que su inercia es la más elevada. El grupo 3 es el más pequeño y homogéneo de los tres. Los cuadros siguientes contienen la descripción de los mismos (ver Tabla 7). 
Tabla 7. Caracterización del Grupo 1. Variables Continuas

\begin{tabular}{|c|c|c|c|c|c|}
\hline Variables & $\begin{array}{l}\text { Media del } \\
\text { grupo }\end{array}$ & Media general & $\begin{array}{l}\text { Desviación } \\
\text { típica del grupo }\end{array}$ & $\begin{array}{l}\text { Desviación } \\
\text { típica general }\end{array}$ & Valor Test \\
\hline No puedo salir a la calle sin móvil, si se me olvida, vuelvo a por él. & 3,039 & 2,519 & 1,202 & 1,373 & 6,07 \\
\hline $\begin{array}{l}\text { Los mayores se enfadan conmigo porque le presto más atención al } \\
\text { móvil que a ellos. }\end{array}$ & 2,704 & 2,288 & 1,105 & 1,268 & 5,26 \\
\hline $\begin{array}{l}\text { Suelo estar mirando el móvil aunque esté con gente, familia o } \\
\text { amigos. }\end{array}$ & 3,020 & 2,637 & 1,029 & 1,259 & 4,86 \\
\hline Por estar usando el móvil duermo menos. & 2,816 & 2,419 & 1,320 & 1,398 & 4,54 \\
\hline $\begin{array}{l}\text { Aunque quisiera no podría prescindir de mi móvil. Si me lo quitaran } \\
\text { en casa me pondría furioso. }\end{array}$ & 2,724 & 2,374 & 1,193 & 1,319 & 4,25 \\
\hline $\begin{array}{l}\text { Tengo la necesidad de estar mirando el móvil continuamente por si } \\
\text { hay algún mensaje nuevo. }\end{array}$ & 2,743 & 2,419 & 1,173 & 1,333 & 3,89 \\
\hline $\begin{array}{l}\text { Me disgusta tener que apagar el móvil en clase, en comidas } \\
\text { familiares, en actos sociales, cine, etc. }\end{array}$ & 2,204 & 1,922 & 1,126 & 1,179 & 3,83 \\
\hline $\begin{array}{l}\text { Me agobio mucho si no tengo el móvil y pienso que alguien que } \\
\text { alguien quiere comunicarse conmigo. }\end{array}$ & 2,796 & 2,497 & 1,199 & 1,386 & 3,45 \\
\hline $\begin{array}{l}\text { Sería la persona más feliz del mundo si me regalaran un móvil de } \\
\text { última generació. }\end{array}$ & 2,882 & 2,591 & 1,251 & 1,433 & 3,24 \\
\hline $\begin{array}{l}\text { Cuando me siento mal, comienzo a usar el móvil y me siento me } \\
\text { siento mucho mejor. }\end{array}$ & 2,678 & 2,387 & 1,254 & 1,467 & 3,17 \\
\hline Me siento más feliz cuanto estoy usando el móvil. & 2,487 & 2,258 & 1,106 & 1,273 & 2,88 \\
\hline
\end{tabular}

\section{Grupo 1. "Los conectados"}

El primer grupo presenta, para 11 de los 21 ítems presentes en la escala de nomofobia, valores medios superiores respecto al total de la muestra, situándose alrededor del punto central de la escala. Aunque no indican un grado muy elevado de nomofobia, muestran dependencia hacia el móvil, no pueden prescindir de él, ni salir a la calle sin su terminal, se agobian si no lo tienen a su lado al creerse incomunicados. Están siempre atentos a su Smartphone, aunque estén con familia y se enfaden con ellos por este motivo. Duermen menos por utilizar el móvil y les disgusta apagarlo en el colegio o reuniones familiares. Creen que su felicidad depende de la posesión de un Smartphone de última generación, o simplemente, de la utilización de un móvil con conexión a Internet. Los estudiantes de este grupo utilizan siempre el teléfono móvil, y tienen entre sus posesiones personales ordenador de mesa y tableta. Casi el $27 \%$ se encuentra en cuarto de la ESO. Lo que más suelen publicar en Internet son fotografías tanto individuales como de grupo, así como selfies.

Les encanta usar Instagram, Whastapp y Twitter, conectándose a estas aplicaciones, y publicando contenido en ellas, en cualquier lugar, incluso en el colegio, y momento del día. Suelen estar conectados al WhatsApp entre 2 y 4 horas diarias, y lo hacen por diversión. Utilizan Twitter para cotillear y están en esta red social menos de una hora al día. En cambio, en Instagram el tiempo diario de conexión sube a más de cinco horas, y lo hacen para sentirse integrados y también por cotilleo. Otra red por la que muestran una preferencia elevada es Snapchat, la usan en cualquier momento y lugar, con una frecuencia diaria de 2 a 3 horas.

Usan también Facebook, Periscope, Ask, Google+ y Youtube. En el caso de Facebook se conectan y publican siempre que pueden o dos o tres veces al día, como media, están dentro de esta red menos de una hora al 
día, utilizándola fundamentalmente para cotillear. Entran en Youtube de tres a diez veces al día, con una permanencia de unas dos o tres horas diarias en total, y lo hacen en cualquier lugar, publicando en esta red social, preferentemente, por la noche. A Periscope se conectan en su casa o en casas de amigos, o zonas de wifi gratis, en cambio Ask es una red social que suelen utilizar en el instituto (ver Tabla 8).

\section{Grupo 2. "Los Creativos"}

El $60 \%$ de los componentes de este grupo son chicos, presentado para todos los ítems de la escala de nomofobia, excepto uno, valores medios inferiores respecto al resto de la muestra y, por tanto, más cercanos al desacuerdo con esos enunciados. Sin embargo, los valores test observados en todos estos casos son negativos, por lo que esto no les caracteriza, en cambio, la percepción que tienen de sí mismos como personas creativas e imaginativas sí.

El 64\% de los estudiantes de este segmento afirma que en su tiempo libre nunca están ociosos sin hacer nada, unos suelen ir a clases particulares después del instituto (6\%), otros van a clases de inglés (9\%), mientras que un $16 \%$ afirma que sus resultados académicos son muy buenos. El 53\% usan poco el móvil, alguna vez como mucho. Les gusta Instagram y Whatsapp, pero no los utilizan con frecuencia. En ambas redes suelen estar menos de una hora diaria, conectándose de 3 a 10 veces al día, en el caso de WhatsApp en concreto, publican por la mañana, en la sobremesa y por la tarde. No les gusta Snapchat ni Ask, y no los utilizan. En cambio si entran en Pinterest y Youtube, en este último caso suelen conectarse dos o tres veces al día lo que implica menos de una hora diaria, preferentemente por la tarde y lo hacen por diversión (ver Tabla 9).

\section{Grupo 3. “Los nomofóbicos"}

El más pequeño de todos con 45 participantes y un comportamiento muy homogéneo según sus inercias. La respuesta de este grupo a la escala de nomofobia presenta unos valores considerablemente superiores respecto al total de la muestra en todos los ítems, siendo por tanto los que muestran una nomofobia más severa, o un comportamiento hacia el móvil más obsesivo.

No saben que hacer sin el móvil, se sienten más felices y seguros con él, primando su uso frente a la relaciones personales, sociales y familiares, mostrando enfado o incomodidad cuanto no están conectados a la red o no pueden comunicarse con los demás a través de su terminal. Se ponen nerviosos si se quedan sin datos o batería, o pierden la señal wifi, nunca salen a la calle sin el teléfono en su mano. El uso continuado del móvil les despista de las tareas escolares e incluso sacrifican horas de sueño por no perder la conexión. Suelen tener discusiones con familia y amigos por la excesiva atención que le prestan a sus smartphones.

Por su forma de relacionarse con los demás, los componentes de este grupo se consideran graciosos, populares y atractivos, con valores por encima de la media general, y esto es algo que les caracteriza. En su tiempo libre les gusta "no hacer nada", suelen estudiar solo en fecha de exámenes. Les encanta WhatsApp, Instagram, Snapchat, Musically y Ask. Se conectan a WhatsApp siempre que pueden, más de 5 horas al día, lo suelen hacer en el colegio y en sitios con wifi gratis. Utilizan esta red de mensajería instantánea para cotillear y sentirse más felices. En cuanto a Instagram, entran en la red siempre que pueden, más de cinco horas al día, preferentemente al medio día y el lugar desde el que se conectan es diverso, el instituto, en casa de amigos o en sitios con wifi gratis. Los motivos por los que usan con tanta intensidad esta red social son variados, afirman que lo hacen para ser felices, conocer gente nueva, por "postureo", para cotillear y porque es lo que se lleva.

Snapchat es otra red muy popular entre los estudiantes de este grupo, se conectan a ella siempre que pueden, con una frecuencia diaria de entre dos y cuatro horas. Suelen entrar en esta red desde casa, en la hora de la sobremesa, y la utilizan porque está de moda, por "postureo", para sentirse más felices, para conocer gente y descubrir cosas nuevas.

Respecto a Musically, como en el caso de las redes ya comentadas, se conectan siempre que pueden y en cualquier momento del día. Lo hacen porque se lleva, para conocer gente y descubrir cosas nuevas, para cotillear, por "postureo", también para evitar el aburrimiento y sentirse mejor. Suelen colgar videos musicales propios. Ninguno de los miembros de este segmento tiene Pinterest. Twitter y Youtube les dejan indiferentes. Entran en Facebook al medio día y lo hacen por diversión, también algunos se conectan a Periscope dos o tres veces al día. 
IRENE RAMOS-SOLER, CARMEN LÓPEZ-SÁNCHEZ Y Ma CARMEN QUILES-SOLER

Tabla 8. Caracterización del Grupo 2. Variables Continuas

\begin{tabular}{|c|c|c|c|c|c|}
\hline Variables & $\begin{array}{l}\text { Media del } \\
\text { grupo }\end{array}$ & Media general & $\begin{array}{l}\text { Desviación } \\
\text { típica del grupo }\end{array}$ & $\begin{array}{l}\text { Desviación } \\
\text { típica general }\end{array}$ & Valor Test \\
\hline Creativo/imaginativo & 4,977 & 4,737 & 1,760 & 1,758 & 2,48 \\
\hline Sociable & 5,126 & 5,358 & 1,581 & 1,516 & $-2,78$ \\
\hline Me molesto cuando quiero buscar algo desde mi móvil y no puedo. & 2,331 & 2,731 & 1,108 & 1,186 & $-6,12$ \\
\hline $\begin{array}{l}\text { Mis amigos se enfadan conmigo porque le hago más caso al móvil } \\
\text { que a ellos. }\end{array}$ & 1,280 & 1,621 & 0,541 & 0,950 & $-6,52$ \\
\hline $\begin{array}{l}\text { Me despisto con las tareas del colegio/instituto por estar mirando y } \\
\text { utilizando mi móvil. }\end{array}$ & 1,766 & 2,220 & 0,960 & 1,225 & $-6,74$ \\
\hline Cuando no tengo wifi en mi teléfono móvil me pongo furioso. & 1,800 & 2,239 & 0,956 & 1,145 & $-6,96$ \\
\hline $\begin{array}{l}\text { A veces pienso que se me va a acabar la batería del móvil y me } \\
\text { pongo nervioso. }\end{array}$ & 1,874 & 2,401 & 1,140 & 1,371 & $-6,97$ \\
\hline $\begin{array}{l}\text { Si me quedo sin wifi, inmediatamente busco señal para conectarme } \\
\text { donde sea. }\end{array}$ & 1,674 & 2,210 & 0,945 & 1,263 & $-7,69$ \\
\hline Me siento más feliz cuanto estoy usando el móvil & 1,697 & 2,258 & 0,971 & 1,273 & $-8,00$ \\
\hline Me da mucho miedo quedarme sin datos en mi móvil. & 1,337 & 1,903 & 0,745 & 1,217 & $-8,45$ \\
\hline $\begin{array}{l}\text { Cuando me siento mal, comienzo a usar el móvil y me siento mucho } \\
\text { mejor. }\end{array}$ & 1,697 & 2,387 & 1,197 & 1,467 & $-8,54$ \\
\hline
\end{tabular}

Fuente: Elaboración propia

Tabla 9. Caracterización del grupo 3. Variables continuas

\begin{tabular}{|c|c|c|c|c|c|}
\hline Variables & $\begin{array}{l}\text { Media del } \\
\text { grupo }\end{array}$ & Media general & $\begin{array}{l}\text { Desviación } \\
\text { típica del grupo }\end{array}$ & $\begin{array}{l}\text { Desviación } \\
\text { típica general }\end{array}$ & Valor Test \\
\hline Creativo/imaginativo & 4,977 & 4,737 & 1,760 & 1,758 & 2,48 \\
\hline Sociable & 5,126 & 5,358 & 1,581 & 1,516 & $-2,78$ \\
\hline Me molesto cuando quiero buscar algo desde mi móvil y no puedo. & 2,331 & 2,731 & 1,108 & 1,186 & $-6,12$ \\
\hline $\begin{array}{l}\text { Mis amigos se enfadan conmigo porque le hago más caso al móvil } \\
\text { que a ellos. }\end{array}$ & 1,280 & 1,621 & 0,541 & 0,950 & $-6,52$ \\
\hline $\begin{array}{l}\text { Me despisto con las tareas del colegio/instituto por estar mirando y } \\
\text { utilizando mi móvil. }\end{array}$ & 1,766 & 2,220 & 0,960 & 1,225 & $-6,74$ \\
\hline
\end{tabular}

Fuente: Elaboración propia 
Tabla 9. Caracterización del grupo 3. Variables continuas (continuación)

\begin{tabular}{|c|c|c|c|c|c|}
\hline Variables & $\begin{array}{l}\text { Media del } \\
\text { grupo }\end{array}$ & Media general & $\begin{array}{l}\text { Desviación } \\
\text { típica del grupo }\end{array}$ & $\begin{array}{l}\text { Desviación } \\
\text { típica general }\end{array}$ & $\begin{array}{l}\text { Valor } \\
\text { Test }\end{array}$ \\
\hline Cuando no tengo wifi en mi teléfono móvil me pongo furioso. & 1,800 & 2,239 & 0,956 & 1,145 & $-6,96$ \\
\hline $\begin{array}{l}\text { A veces pienso que se me va a acabar la batería del móvil y me } \\
\text { pongo nervioso. }\end{array}$ & 1,874 & 2,401 & 1,140 & 1,371 & $-6,97$ \\
\hline $\begin{array}{l}\text { Si me quedo sin wifi, inmediatamente busco señal para conectarme } \\
\text { donde sea. }\end{array}$ & 1,674 & 2,210 & 0,945 & 1,263 & $-7,69$ \\
\hline Me siento más feliz cuanto estoy usando el móvil & 1,697 & 2,258 & 0,971 & 1,273 & $-8,00$ \\
\hline Me da mucho miedo quedarme sin datos en mi móvil. & 1,337 & 1,903 & 0,745 & 1,217 & $-8,45$ \\
\hline $\begin{array}{l}\text { Cuando me siento mal, comienzo a usar el móvil y me siento mucho } \\
\text { mejor. }\end{array}$ & 1,697 & 2,387 & 1,197 & 1,467 & $-8,54$ \\
\hline
\end{tabular}

Fuente: Elaboración propia

\section{DISCUSIÓN Y CONCLUSIONES}

\section{CONCLUSIONES}

El estudio de la nomofobia, el uso/abuso de los teléfonos móviles y la conexión a las redes sociales a través de estos dispositivos entre adolescentes es un tema actual y pertinente en el ámbito de la comunicación, la educación y salud. Tras la revisión teórica y el trabajo de campo efectuado en esta investigación se observa que estamos antes una adicción comportamental con un impacto real en el ámbito educativo, social y familiar que puede provocar problemas personales y/o sociales.

La mayoría de las investigaciones que han evaluado y realizado la medición sobre el uso problemático de los teléfonos inteligentes, denominan de forma diferente el mismo problema (Simó et al., 2017), perteneciendo la mayoría de ellas al ámbito anglosajón, cuyo entorno sociocultural presenta diferencias evidentes con la realidad cultural española.

El objetivo de esta investigación era adaptar y validar la escala de nomofobia propuesta por Yildirim y Correia (2015), a los estudiantes españoles de Educación Secundaria Obligatoria, de menor edad y con un entorno cultural y social diferente. Los resultados obtenidos en los análisis estadísticos realizados confirman que dicha adaptación transcultural es válida y fiable. Los ítems de la escala han mostrado una buena consistencia interna, generando puntuaciones fiables.

Tras la aplicación del cuestionario a los estudiantes españoles, se observa, que la respuesta a la escala muestra una importante limitación, las respuestas suelen ser "socialmente deseables" y parciales.

Tanto en el primer grupo o segmento como en el tercero, a pesar de que los valores de la escala de nomofobia se situaban por encima de la media central de la escala, y mostraban su acuerdo de forma general con los enunciados, los valores ilustrativos utilizados para la descripción de las particiones nos han enseñado otra imagen de los estudiantes que refuerza la idea de dependencia de los móviles. Los datos que se han usado para ilustrar y describir las particiones han mostrado un uso abusivo de los teléfonos inteligentes y sus aplicaciones, junto al temor o miedo a estar desconectados.

Estos resultados pueden indicar dos cosas diferentes. La primera de ellas mostraría que los estudiantes han optado por una respuesta de conveniencia "socialmente deseable" y no muy real, en cada uno de los enunciados de la escala de nomofobia. Lo que puede significar no querer reconocer una adicción comportamental. La segunda, es más sencilla y preocupante aún, no creen que realmente tengan un 
problema y asumen su intenso comportamiento digital como algo normal.

Nuestra investigación muestra que las ofertas digitales y el tiempo de uso de los smartphones están influyendo en nuestro estilo de vida. La preocupación es mayor cuando llegamos a hablar de conductas de abuso relacionadas con los más jóvenes. Es una evidencia que las TICs son de una utilidad insustituible, pero vemos necesario y urgente poner en marcha programas educativos que establezcan límites a su utilización y a los tiempos de uso de las mismas. Es necesario dar a conocer las ventajas, pero también los riesgos, intentando que los menores sean más críticos con las tecnologías y esto sirva como elemento de protección ante los posibles efectos negativos.

De los resultados obtenidos en la investigación se plantean algunas limitaciones que, a su vez, pueden ser vistas como futuras investigaciones. Por un lado, el universo de estudio abarcaba únicamente a estudiantes de Educación Superior Obligatoria pero sería interesante observar y validar esta escala a partir de su aplicación a otras franjas de edad, sobre todo en el entorno universitario. Por otro lado, habría que hacer referencia al ámbito geográfico del estudio: Provincia de Alicante. En este sentido, al igual que se puede plantear ampliar la edad del universo de estudio, cabría la posibilidad de llevar el estudio a un área geográfica más amplia, de modo que se pudieran observar posibles diferencias, no solo por edad sino también por lugar de procedencia de los encuestados.

\section{REFERENCIAS}

Arab y Díez (2015). Impacto de las redes sociales e Internet en la adolescencia: aspectos positivos y negativos. Revista Médica Clínica Las Condes, 26(1) 07-13. Disponible en: http://www.elsevier.es/esrevista-revista-medica-clinica-las-condes-202-articulo-impacto-lasredes-sociales-e-S0716864015000048

Bragazzi, N.L.; del Puente, G.A. (2014). A proposal for including nomophobia in the new DSM-V. Psychol Res Behav Manag, 7:155-60. Disponible https://www.ncbi.nlm.nih.gov/pmc/articles/PMC4036142/ doi: $\underline{10.2147 / P R B M . S 41386}$

Christakis et al. (2001). Problematic internet usage in US college students: a pilot study. BMC Med. Jun 22, 9:77. Disponible en: https://bmcmedicine.biomedcentral.com/articles/10.1186/17417015-9-77 doi: 10.1186/1741-7015-9-77.
Comrey, A. L., \& Lee, H. B. (1992). A first course in factor analysis. Psychology Press.

DeVellis, R. F. (2003). Scale development: Theory and applications (Vol. 26). Thousand Oaks, CA: Sage.

Digital Marketing Trends (Ditrendia, 2016). Informe Mobile en España y en el Mundo 2016.2 Disponible en: http://www.amic.media/media/files/file 352 1050.pdf

Espinar, E.; González M.J. (2009). Jóvenes en las redes sociales virtuales. Un análisis exploratorio de las diferencias de género. Feminismo/s, 14, 87-106. Disponible en: https://rua.ua.es/dspace/bitstream/10045/13302/1/Feminismos 1 4 06.pdf

George, D., \& Mallery, P. (2011). SPSS for windows step by step: A simple guide and reference 18.0 update. Pearson.

Gomes, F.; Sendín, J.C. (2016). Internet como refugio y escudo social: Usos problemáticos de la Red por jóvenes españoles. Comunicar [revista en Internet], 22(43), 45-53. Disponible en: http://www.revistacomunicar.com/verpdf.php?numero=43\&art iculo=43-2014-04

Hambleton, RK. (1994). Guidelines for adapting educational and psychological tests: a progress report. European Journal of Psychological Assessment, 10 (3), 229-244.

Interactive Advertisising Boureau Spain (IAB) (2016). Estudio Anual de Redes Sociales. Disponible en: http://www.iabspain.net/wpcontent/uploads/downloads/2016/04/IAB_EstudioRedesSociales_2 016 VCorta.pdf

King, A. L. S., Valença, A. M., \& Nardi, A. E. (2010). Nomophobia: The mobile phone in panic disorder with agoraphobia: Reducing phobias or worsening of dependence? Cognitive and Behavioral Neurology, 23(1), 52-54. En Yildirim, C.; Correia, A.P. (2015). Exploring the dimensions of nomophobia: Development and validation of a selfreported questionnaire. Computers in Human Behavior, 49, 130-137. Disponible

en: http://www.sciencedirect.com/science/article/pii/S0747563215 $\underline{001806}$

SecurEnvoy (2012). 66\% of the population suffer from nomophobia the fear of being without their phone. Disponible en https://www.securenvoy.com/blog/2012/02/16/66-of-thepopulation-suffer-from-nomophobia-the-fear-of-being-withouttheir-phone/

Simó Sanz, C.; Martínez Sabater, A.; Ballester Tarín, M.L; Domínguez Romero, A. (2017). Instrumentos de evaluación del uso problemático del teléfono móvil/Smartphone. Health and Addictions, 17 (1), 5-14. Disponible en: http://ojs.haaj.org/index.php/haaj/article/view/265/274

Suriá, R. (2015). Jóvenes con discapacidad motora y redes sociales online, ¿nuevos espacios para el desarrollo de habilidades sociales? Anuario de Psicología, 45(1), 71-85.

Tabachnick, B. G., \& Fidell, L. S. (2013). Using multivariate statistics. Boston: Pearson. 
ADAPTACIÓN Y VALIDACIÓN DE LA ESCALA DE NOMOFOBIA DE YILDIRIM Y CORREIA EN ESTUDIANTES ESPAÑOLES DE LA EDUCACIÓN SECUNDARIA...

Tresáncoras, A.G.; García-Oliva, C.; Piqueras, J.A. (2017). Relación del uso problemático de whatsapp con la personalidad y la ansiedad en adolescentes. Health and Addictions, 17 (1), 27-36. Disponible en: http://ojs.haaj.org/index.php/haaj/article/view/272/276

Walsh, S. P., White, K. M., \& Young, R. M. (2010). Needing to connect: The effect of self and others on young people's involvement with their mobile phones. Australian Journal of Psychology, 62(4), 194-203. 